\title{
RELACIÓN ENTRE LA SEGREGACIÓN DE GÉNERO EN LAS DISCIPLINAS DE ESTUDIO UNIVERSITARIAS Y EL EMPLEO DE LAS PERSONAS GRADUADAS EN COSTA RICA
}

\author{
Laura C. Blanco ${ }^{1}$
}

Recibido: 17/03/2017

Aprobado: 17/02/2018

RESUMEN

Se utilizaron datos de las encuestas a las personas graduadas de las universidades costarricenses entre 2000 y 2010 para examinar si la segregación por género de las carreras universitarias se asociaba con la probabilidad de estar empleada. Los resultados mostraron que las personas graduadas que estudiaron carreras dominadas por hombres tuvieron una mayor probabilidad de estar empleadas. No obstante, mientras las mujeres que ingresaron a programas predominantemente masculinos poseyeron una ventaja en el empleo, los hombres que incursionaron en carreras femeninas fueron penalizados por ello. La educación, experiencia laboral profesional y diferencias de clase resultaron también significativas para comprender el empleo femenino, aunque en menor grado para los hombres. Finalmente, una descomposición Oaxaca-Blinder para los modelos no lineales mostró que únicamente cerca de un cuarto de la brecha en el empleo se explicaba por las diferencias en las características individuales, sugiriendo la existencia de discriminación a favor de los hombres.

PALABRAS CLAVE: LABORAL, CONDICIONES DE EMPLEO

CLASIFICACIÓN JEL: E12, E31, E52

\section{ABSTRACT}

This paper used data from surveys of recent graduates from Costa Rican universities during the period 2000-2010 to test whether sex-typing of subject fields and areas of study were associated with the probability of being employed. The findings indicated that graduates who studied male-dominated subject fields were more likely to be employed. However, while women who entered predominantly male fields of study had an employment advantage, men who transgressed into female fields of study seem to be punished for it. Schooling, job experience and class differences were also significant in explaining female employment, but less so for men. Finally, an Oaxaca-Blinder

1 Universidad de Costa Rica, Escuela de Economía; Código postal 11501-2060; San José, Costa Rica; lauracristina.blanco@ucr.ac.cr 
decomposition for nonlinear models showed that only about a quarter of the gender employment gap was explained by differences in individual characteristics, suggesting the existence of discrimination in favor men.

KEYWORDS: LABOR, CONDITIONS OF EMPLOYMENT.

JEL CLASIFICATION: E12, E31, E52

\section{INTRODUCCIÓN}

La segregación ocupacional ha sido identificada como una de las barreras más significativas para cerrar la brecha de ingresos entre los géneros ${ }^{2}$, mientras que la menor participación femenina en el mercado laboral ${ }^{3}$ se explica principalmente por una menor experiencia laboral y las mayores tasas de desempleo femenino responden a las decisiones reproductivas $y$ a factores institucionales ${ }^{4}$. Las mujeres cuentan con una mayor probabilidad de estar desempleadas que los hombres (Organización Internacional del Trabajo, 2014) y reciben menores incrementos salariales debido a su intermitencia en el empleo (Manning \& Swaffield, 2008), la cual se asocia también a sus decisiones reproductivas. Estos resultados apoyan las teorías de Becker sobre capital humano (1962), división del trabajo (1991) y discriminación (1971): los diferenciales de género en los resultados laborales están claramente marcados por las decisiones de las mujeres relativas a la cantidad y calidad de su inversión en educación, así como su grado de especialización en el hogar y en el mercado laboral.

No obstante, la brecha de género permanece mayoritariamente sin ser explicada: el coeficiente de discriminación representa la mayor parte de la brecha de género en el ingreso (Oaxaca, 1973; Mumford \& Smith, 2009) y en el empleo (Ortega Masagué, 2008; Nagore-García, 2015), aunque esta última es poco estudiada. Asimismo, las mujeres profesionales se ven afectadas, no sólo por la segregación ocupacional y la discriminación, sino también por la segregación de género asociada a sus disciplinas y áreas de estudio, las cuales son significativas al explicar los diferenciales de género en los resultados laborales ${ }^{5}$. Las brechas de género en una profesión son dependientes del grado en que la entrada de mujeres afecte el estatus jerárquico de esa profesión (Goldin, 2002), de forma que las profesiones con credencialización o mecanismos de certificación, paridad educativa, mayor participación laboral femenina y homogeneidad demográfica entre mujeres y hombres podrían mostrar menores brechas de género (Goldin \& Katz, 2012).

Aunque existe una amplia y consistente literatura que estudia las brechas de género en el ingreso -incluyendo aquellas entre personas graduadas-, tradicionalmente el énfasis de estudio se ha centrado en países desarrollados, en parte debido a la falta de información en los países en vías de desarrollo. Este artículo contribuye a la literatura al analizar la relación existente entre la segregación de género en las disciplinas de estudio y el empleo en el contexto de un país en vías de desarrollo: Costa Rica.

Costa Rica resulta un caso interesante de analizar debido a que está marcado por amplios contrastes: a pesar de ser un país de ingresos medios, con un producto per capita de aproximadamente 14.000 dólares, una quinta parte de su población vive bajo la línea de pobreza y su coeficiente de Gini es de 0.49, ubicándose entre los países de mayor inequidad en América Latina. Costa Rica es también un Estado católico por constitución, con una agenda de género e igualdad sexual defi-

2 Becker, 1971; Oaxaca, 1973; Rosenfeld, 1979; Walby, 1988; Blau, 1999; Goldin, 2002; Miech, Eaton y Liang, 2003; Blau y Kahn, 2007; Mumford y Smith, 2009.

3 Heckman y Willis, 1977; Goldin, 1983; Blau y Kahn, 2007.

4 Ahn y Mira, 2002; Adsera, 2005; Azmat, Güell y Manning, 2006.

5 Machin y Puhani, 2002, 2005; Black, Haviland, Sanders y Taylor, 2008; Chevalier, 2011; Zafar, 2013. 
ciente en comparación con los países desarrollados: el aborto, el matrimonio para personas del mismo género, la anticoncepción para adolescentes y la anticoncepción de emergencia están prohibidas; la educación sexual obligatoria apenas fue establecida en 2013; la fertilización in vitro se aprobó en 2016 como respuesta a un fallo de la Corte Interamericana de Derechos Humanos; menos de la mitad de las mujeres participan en la fuerza laboral; sólo $2 \%$ de las personas empleadoras son mujeres; únicamente 15\% de las empresas colocan a mujeres en puestos gerenciales y el desempleo femenino es de 11\%, 4.3 puntos porcentuales (p.p.) por encima del masculino (Banco Mundial, 2015). Las mujeres costarricenses también experimentan una mayor duración en el desempleo y son más proclives a moverse hacia la inactividad que los hombres (Castro, Garita \& Odio, 2014).

En contraste con lo anterior, Costa Rica es uno de los países que experimentaron una reversión educativa en la década de los noventa: hoy en día, los muchachos tienen 1.3 veces más probabilidad que las muchachas de ser repitentes en secundaria, se cuenta con paridad educativa en primaria y secundaria y las mujeres tienen 1.24 veces más probabilidad de matricularse en la educación superior (Banco Mundial, 2015). Y ya se muestran algunos signos de que esta reversión educativa pueda estar impactando el mercado laboral: mientras los hombres ganan, en promedio, cerca de $24 \%$ más que las mujeres (Cedeño, González \& Pizarro, 2015), estas ganan más que los hombres en los percentiles más altos debido a su ventaja en capital humano y características individuales, a pesar de la persistencia del coeficiente de discriminación a lo largo de toda la distribución salarial (Rodríguez \& Segura, 2015). Es probable, por tanto, que los cambios observados en lo más alto de la distribución estén siendo motivados por la reversión de la educación superior: conforme la proporción de mujeres dentro de las personas graduadas universitarias aumente, las mujeres podrían ir ganando mayor acceso a mejores puestos y salarios. Este artículo investiga la relación existente entre la segregación de género en las áreas de estudio y el nivel de empleo, en un contexto en que las mujeres representan la mayoría de personas graduadas universitarias en un mercado marcado por niveles significativos de discriminación en contra de las mujeres, tal y como lo es el mercado costarricense.

El artículo está estructurado de la siguiente forma: la sección metodológica describe la base de datos y provee las estadísticas descriptivas para la muestra balanceada utilizada en el análisis, así como los métodos utilizados en las estimaciones. La sección de resultados presenta las estimaciones de una regresión logística sobre la probabilidad de encontrar empleo, así como su respectiva descomposición de Oaxaca-Blinder para modelos no lineales. Finalmente, la última sección concluye.

\section{METODOLOGÍA}

\section{Base de datos}

En 2013, el Consejo Nacional de Rectores (CONARE) publicó la encuesta Seguimiento de la condición laboral de las personas graduadas 2000-2007 de las universidades costarricenses (CONARE, 2013), en donde recolecta información sobre el grado obtenido, la universidad de estudio, la experiencia de estudio, las condiciones actuales de empleo, las razones de desempleo e información sobre el contexto familiar y estudios secundarios de quienes recientemente se graduaron de las instituciones públicas y privadas. La encuesta se realizó entre el 7 de junio y el 8 de noviembre de 2010 y recolectó información de 12412 personas que obtuvieron un título de bachillerato o licenciatura entre 2000 y 2007. En 2013, CONARE implementó una encuesta similar (Seguimiento de la condición laboral de las personas graduadas 2008-2010 de las universidades costarricenses) en la que recolectó información sobre quienes obtuvieron su título entre 2008 y 2010 y la publicó en 2015. Esta segunda encuesta contiene información de 12300 personas. En total, ambas encuestas recolectaron datos para 24712 personas. En ambos casos el diseño muestral es representativo de 
la población graduada y está estratificado por disciplina de estudio y sector universitario (público o privado).

Lamentablemente, aunque estas encuestas proveen información valiosa sobre las personas graduadas universitarias en Costa Rica, dicha información es considerablemente limitada: no se pidió reportar el monto del salario ni las horas trabajadas (i.e., no es posible estimar el salario por hora a partir de esta encuesta), a quienes cuentan con varios empleos no se les solicitó información para cada trabajo en forma separada, tampoco se preguntó por el historial laboral, esfuerzos para buscar empleo, años invertidos en educación o decisiones reproductivas, que son fundamentales para entender el mercado laboral. Debido a estas limitaciones del diseño de la encuesta, el artículo debió enfocarse en estudiar el empleo en lugar del desempleo: a las personas encuestadas se les preguntó si se encontraban laborando actualmente, de manera que este grupo es identificable. A aquellas personas no empleadas se les preguntó por las razones por las que no trabajan. Esto permitió identificar a aquellas personas que se encontraban fuera de la fuerza de trabajo porque todavía estaban estudiando, no querían trabajar o estaban retiradas. Sin embargo, como a quienes no se encontraban trabajando no se les preguntó si habían buscado trabajo recientemente, no fue posible distinguir a quienes se encontraban en desempleo de quienes se habían retirado de la fuerza laboral, salvo por los tres casos mencionados con anterioridad. Por simplicidad, quienes no se encontraban trabajando, ni estudiando, ni estaban en su jubilación, ni expresaron no querer trabajar se consideran como personas desempleadas en el resto del documento, aunque esta es solo una estimación aproximada.

Debido a que la población de estudio se refiere a personas en la fuerza laboral, se excluye de la muestra las personas que vivían fuera de Costa Rica, todavía estudiaban, no deseaban trabajar o se encontraban pensionadas. En forma similar, las personas con ítems sin responder o respuestas incoherentes también fueron excluidas, lo que deja una muestra balanceada de 22308 personas, $61.9 \%$ de las cuales corresponden a mujeres y 38.1\% a hombres ${ }^{6}$. La información presentada utiliza los correspondientes ponderadores, de forma que los datos son representativos de la población graduada.

\section{Descripción de variables}

La tabla 1 presenta las estadísticas descriptivas de las variables de interés en la muestra. Dichas estadísticas también se presentan en forma separada según género. Como es de esperar, la tasa de empleo es alta (97\%) entre las personas graduadas, aún más para los hombres (98.2\%), con un diferencial de empleo de 1.89 p.p. a favor de ellos.

La base de datos provee información detallada sobre el tipo de capital humano en que cada persona invirtió. La muestra contiene información de 109 disciplinas de estudio agrupadas en 8 áreas: humanidades, ciencias, disciplinas económicas, ciencias sociales, educación, recursos naturales, ingeniería y ciencias de la salud. La tabla 1 muestra diferencias por género en las preferencias por las disciplinas de estudio: mientras la mayoría de mujeres y hombres se gradúan de educación o alguna disciplina económica, las mujeres se encuentran altamente concentradas en la primera (39.2\%). En contraste, los hombres se encuentran más dispersos entre las diferentes disciplinas. La segregación de género resulta más obvia al observar la distribución de género a lo interno de cada área de estudio: las mujeres representan el 79\% de todas las personas graduadas en educación y casi el $70 \%$ en ciencias sociales y ciencias de la salud, mientras que los hombres representan más del $70 \%$ de las personas graduadas en ciencias e ingeniería.

6 Esta muestra es representativa de la población. Como las mujeres tienen una mayor probabilidad de asistir a la universidad en Costa Rica, $62,7 \%$ de todas las personas graduadas son mujeres. 
Siguiendo a Hakim (2002) y Sullivan, Joshi y Leonard (2008), se creó una variable con tres categorías para clasificar la segregación de género en las disciplinas de estudio. Aquellas disciplinas en donde las mujeres representan menos del $40 \%$ se consideran dominadas por hombres, aquellas cercanas a la paridad, con representación femenina entre $40 \%$ y menos de $60 \%$, se consideran integradas y aquellas disciplinas en donde las mujeres representan $60 \%$ o más de las personas graduadas son consideradas predominantemente femeninas. Esta clasificación difiere de la de Gutiérrez y Kikut (2014, p.11-13) en su resumen descriptivo de las bases de datos ${ }^{7}$. La clasificación de las disciplinas de estudio aquí utilizada se muestra en el Anexo 1. Hay 19 disciplinas de estudio predominantemente masculinas, las cuales incluyen algunas de las humanidades (filosofía y teología), física, educación musical, educación física, disciplinas asociadas a agronomía, geografía y la mayoría de las ingenierías. Por otro lado, 53 disciplinas se clasificaron como femeninas. Estas incluyen las humanidades asociadas a las artes y las lenguas, biología, algunas de las ciencias sociales (psicología, sociología, trabajo social, etc.), las ciencias de la salud (enfermería, farmacia, optometría, etc.) y la mayoría de las disciplinas en educación. Las restantes 37 disciplinas se consideran integradas e incluyen a la mayoría de las disciplinas económicas.

La tabla 1 muestra que la mayoría de mujeres (63.7\%) se gradúan de una disciplina predominantemente femenina. Asimismo, las mujeres son más reticentes a incursionar en disciplinas predominantemente masculinas que los hombres, en disciplinas femeninas. En términos de elección, esto puede sugerir que la segregación de disciplinas es, mayoritariamente, un reflejo de las elecciones de carrera de las mujeres más que de los hombres. No solo las mujeres son más numerosas, parecen estar reforzando las esferas separadas al optar por disciplinas en donde se observa una mayor segregación. Por el contrario, el hecho de que los hombres se encuentren dispersos a lo largo de las diferentes categorías también sugiere que la segregación podría no estar tan enraizada en ellos. De hecho, el índice de disimilitud ${ }^{8}$ para las disciplinas de esta muestra es de $31 \%$, lo que significa que $31 \%$ de las mujeres (u hombres) tendrían que cambiar de disciplina de estudio para alcanzar una representación igualitaria en todas las disciplinas. Por tanto, podría argumentarse que la disparidad a lo largo de las disciplinas de estudio es relativamente moderada.

La base de datos también provee información sobre el tipo de grado obtenido, el cual sirve de proxy para el nivel de inversión en capital humano. De acuerdo a la base de datos, la mitad de las personas encuestadas poseía un título de licenciatura al momento de la encuesta, el cual puede interpretarse como un año adicional de educación, ya que la licenciatura usualmente consiste en un programa de un año después del bachillerato. Únicamente cerca de un tercio de las personas encuestadas se graduó de una de las cuatro universidades públicas, lo cual no es ninguna sorpresa, dado que existen 53 universidades privadas en Costa Rica. Y, 53\% de las personas en la muestra luego continuó sus estudios y obtuvo un segundo grado universitario. De estas, $45 \%$ obtuvo un posgrado.

Lamentablemente, la encuesta no indaga sobre el historial laboral de las personas, por lo que no se cuenta con información exacta sobre los años de experiencia laboral ${ }^{9}$. Como es usual en la literatura sobre capital humano y siguiendo a Mincer (1974), se procedió a sustituir esta variable con la experiencia laboral potencial, la cual cuantifica los años transcurridos desde la finalización

7 Gutiérrez y Kikut (2014) solo clasifican 36 disciplinas en tres categorías: aquellas en donde los hombres representan más del 85\% de las graduadas, aquellas en donde las mujeres representan más del $85 \%$ de las graduadas y aquellas en donde mujeres y hombres representan menos del $55 \%$ de las graduadas.

8 El índice de disparidad (Darity Jr. \& Mason, 1998; y Blau, 1999) se estima como d=1/2 $\sum_{i}\left(\left|\mathrm{f}_{i}-\mathrm{m}_{i}\right|\right)$, donde $\mathrm{f}_{i}$ es el porcentaje de mujeres en la disciplina $i$ y $m_{\mathrm{i}}$ es el porcentaje de hombres en $i$.

9 La encuesta sí pregunta por el tiempo que la persona tiene en su empleo actual, pero las respuestas son inconsistentes con la edad de las personas graduadas e historia de capacitación formal. Lo anterior plantea dudas sobre la calidad de la información relacionada con esta pregunta. Por tanto, se decidió no utilizar dicha pregunta y aproximar la experiencia profesional potencial a partir del año de graduación de la universidad. 
de los estudios y hasta la fecha de la encuesta. Ahora bien, debido a que, en este caso, la población estudiada es universitaria, esta variable muestra la experiencia laboral profesional potencial, i.e., los años transcurridos tras la graduación durante los cuales la persona pudo haber ejercido como profesional. En promedio, las personas en la muestra contaban con cinco años de experiencia potencial como profesionales al ser encuestadas.

Una característica particular de Costa Rica es el extenso período requerido para concluir un programa de estudios en la universidad. Esto puede aproximarse en la encuesta como el período transcurrido entre la graduación de la universidad y la secundaria y es, en promedio, de 11.6 años. Lo anterior contrasta con la duración de cuatro o cinco años usualmente prevista para los programas de bachillerato y licenciatura, respectivamente, $y$ evidencia un problema particular de la economía costarricense que debe ser explorado con más detalle en futuras investigaciones, ya que una considerable cantidad de años productivos se pierden debido a las ineficiencias en el sistema de la educación terciaria. Como es posible que las personas hayan trabajado durante el período en que estudiaban en la universidad, se incluye esta variable como una aproximación de la experiencia laboral potencial no profesional. No obstante, cabe resaltar que debido a que el análisis es sobre profesionales y a que, metodológicamente, la estimación de la experiencia laboral potencial considera únicamente el tiempo transcurrido después de la conclusión de estudios, la variable de interés será la referida a la experiencia laboral profesional potencial. La aproximación de la experiencia laboral no profesional se incluye únicamente para controlar por la particularidad del caso costarricense. Finalmente, ninguna de estas dos variables resulta significativamente diferente entre mujeres $y$ hombres, lo cual probablemente se relacione a la falta de información sobre la historia laboral, ya que se esperaría que las mujeres, en promedio, se ausenten por un tiempo de la fuerza laboral, dado que históricamente son ellas quienes se retiran del mercado laboral para dedicarse a la crianza infantil y cuido de personas en el hogar.

Tampoco se observan diferencias significativas entre mujeres y hombres en las variables que miden el contexto socio-económico anterior. En promedio, cerca de una quinta parte de las personas graduadas asistió a una institución secundaria privada. Esto sugiere que las personas provenientes de colegios privados están sobrerrepresentadas en la población de graduadas universitarias, ya que estas instituciones apenas representan el 11\% de la matrícula en secundaria (Ministerio de Educación Pública, 2016). Lo anterior podría sugerir la existencia de barreras de acceso a la educación terciaria.

El índice de competitividad cantonal (Ulate, Madrigal, Ortega \& Jiménez, 2012) se utilizó como una medida del contexto socio-económico de la persona. Este es un índice que oscila entre 0 y 1 y ordena los cantones de acuerdo a su desarrollo en siete áreas: producción, gobierno, infraestructura, clima de negocios, fuerza laboral, innovación y calidad de vida. La mayoría de las personas encuestadas viven en áreas de desarrollo bajo (48.8\%) o medio (20.5\%) y apenas una de cada cinco vive en áreas de desarrollo alto. Finalmente, los datos también muestran que cerca de la mitad de las personas encuestadas viven actualmente con una pareja. 
TABLA 1

ESTADÍSTICAS DESCRIPTIVAS PARA LA MUESTRA DE PERSONAS GRADUADAS DE LAS UNIVERSIDADES COSTARRICENSES EN EL PERÍODO 2000-2010

\begin{tabular}{|c|c|c|c|}
\hline Muestra según género & Agrupada & Mujeres & Hombres \\
\hline Observaciones & 22308 & 13218 & 9090 \\
\hline \multicolumn{4}{|l|}{ Empleo (en porcentajes) } \\
\hline Graduadas actualmente empleadas & 97.00 & 96.30 & 98.20 \\
\hline Graduadas actualmente desempleadas (proxy) & 3.00 & 3.70 & 1.80 \\
\hline \multicolumn{4}{|l|}{ Calidad del capital humano (en porcentajes) } \\
\hline \multicolumn{4}{|l|}{ Grado por segregación de género: } \\
\hline Programas dominados por hombres & 15.26 & 5.60 & 30.90 \\
\hline Programas integrados & 36.17 & 30.70 & 45.10 \\
\hline Programas dominados por mujeres & 48.57 & 63.70 & 24.00 \\
\hline \multicolumn{4}{|l|}{ Grado por área de estudio: } \\
\hline Humanidades & 2.70 & 2.46 & 3.09 \\
\hline Ciencias & 6.50 & 2.89 & 12.35 \\
\hline Disciplinas económicas & 22.43 & 19.79 & 26.72 \\
\hline Ciencias sociales & 15.54 & 17.56 & 12.30 \\
\hline Educación & 30.79 & 39.22 & 17.12 \\
\hline Recursos naturales & 1.81 & 1.24 & 2.70 \\
\hline Ingeniería & 8.18 & 3.48 & 15.81 \\
\hline Ciencias de la salud & 12.04 & 13.35 & 9.88 \\
\hline Personas graduadas de licenciatura & 50.10 & 51.50 & 47.90 \\
\hline Personas graduadas de universidades públicas & 34.80 & 33.20 & 37.50 \\
\hline Personas con un segundo grado académico & 53.30 & 54.10 & 51.90 \\
\hline \multicolumn{4}{|l|}{ Experiencia profesional potencial (Promedios) } \\
\hline Años entre graduación universitaria y secundaria & 11.60 & 11.50 & 11.80 \\
\hline Años transcurridos tras la gra-duación & 5.30 & 5.20 & 5.50 \\
\hline Promedio del cuadrado de años tras la graduación & 33.20 & 32.15 & 34.78 \\
\hline \multicolumn{4}{|l|}{ Variables de control (en porcentajes) } \\
\hline Asistió a una secundaria privada & 21.50 & 21.80 & 21.10 \\
\hline Vive con una pareja & 50.50 & 49.70 & 51.70 \\
\hline \multicolumn{4}{|l|}{ Desarrollo regional: } \\
\hline Muy bajo & 9.00 & 10.10 & 7.30 \\
\hline Bajo & 48.80 & 48.80 & 48.60 \\
\hline Medio & 20.50 & 20.20 & 20.90 \\
\hline Alto & 13.10 & 12.30 & 14.30 \\
\hline Muy alto & 8.70 & 8.60 & 8.80 \\
\hline Mujeres graduadas & 61.90 & 100.00 & 0.00 \\
\hline
\end{tabular}

Fuente: CONARE $(2013,2015)$. 


\section{Métodos}

Se estimó tres modelos de regresión logit con el fin de determinar si el empleo de las personas graduadas se asocia con la segregación sexual de las carreras universitarias, así como con las áreas de estudio. En cada caso, la probabilidad de estar empleada $\left(\mathrm{e}_{\mathrm{i}}=1\right)$ se deriva del siguiente modelo de variables latentes:

$$
e_{i}^{*}=\gamma x_{i}+\xi_{i}
$$

donde $x$ es la matriz de características individuales, $\gamma$ es el vector de coeficientes y $\xi$ refiere al término de error. Las variables incluidas en x varían en cada modelo.

En el modelo A, se regresó el empleo únicamente sobre las dos variables de interés: una variable categórica que indica si el grado obtenido corresponde a una disciplina predominantemente masculina, integrada o femenina y una variable categórica para el área de estudio. La inclusión de estas variables busca falsar la hipótesis de que la segregación sexual de las carreras y las áreas de estudio tienen repercusiones sobre los resultados laborales de las personas ${ }^{10}$.

El modelo B incluye variables que proveen información adicional sobre el capital humano: tres variables binarias para identificar si la persona encuestada obtuvo un título de licenciatura en lugar de bachillerato, se graduó de una universidad pública y si obtuvo un segundo título; dos variables continuas para aproximar la experiencia profesional potencial medida a través de la diferencia entre la fecha de la entrevista $y$ los años de la graduación universitaria y su valor al cuadrado; $y$ una variable para aproximar la experiencia laboral no profesional, medida como el lapso de tiempo entre la graduación de la universidad y la secundaria. Como es usual en la literatura, la escogencia de estas variables toma como referente el modelo de capital humano de Becker (1962), así como el modelo sobre participación laboral femenina de Heckman (1976). Consistentemente y conforme al modelo de capital humano, la literatura indica que tanto los ingresos como la participación laboral responden a la educación y la experiencia laboral de una persona ${ }^{11}$. En tanto la población aquí estudiada cuenta con educación terciaria, las diferencias en el nivel de educación se miden distinguiendo a aquellas personas que continuaron sus estudios más allá del bachillerato universitario (i.e., obtuvieron una licenciatura o segundo diploma) y mediante posibles diferencias en la calidad de la educación, la cual se mide distinguiendo si la persona asistió a una universidad pública o privada.

Por otra parte, como lo que se desea estimar es la probabilidad de empleo de las personas graduadas de la universidad, se considera la experiencia profesional potencial. Como ya se mencionó, es necesario hacer esta aproximación porque no se cuenta con el historial laboral de las personas, de forma que no se puede calcular la experiencia laboral ni se cuenta con información sobre la cantidad de empleos desde la graduación, como en Chevalier (2011). Si bien esto representa una limitación, es usual en la literatura utilizar como aproximación de la experiencia la experiencia potencial $^{12}$. Finalmente, es necesario reiterar una vez más que la encuesta utilizada cuenta, entre sus deficiencias, con la ausencia de información sobre las decisiones reproductivas de las personas. Esto supone una limitación en los alcances del modelo a estimar, pues desde Heckman (1976) se sabe que las decisiones de participación laboral de las mujeres están fuertemente ligadas a dichas decisiones reproductivas. Desafortunadamente, la encuesta no ofrece ninguna variable que pueda servir como proxy para solventar esta deficiencia y es probable que parte del efecto asociado a las decisiones reproductivas esté siendo captado por los coeficientes de tener pareja y ser mujer.

10 Véase Hakim, 2002; Machin y Puhani, 2002, 2005; Sullivan, et al., 2008; Black, et al., 2008; Chevalier, 2011; Goldin y Katz, 2012; y Zafar, 2013.

11 Becker, 1971; Oaxaca, 1973; Oaxaca y Ransom, 1994; Miech, et al., 2003; Azmat, et al., 2006; Blau y Kahn, 2007; Black, et al., 2008; Ortega Masagué, 2008; Mumford y Smith, 2009; Castro, et al., 2014; Cedeño, et al., 2015; Nagore-García, 2015; Rodríguez y Segura, 2015. 
Por su parte, el modelo C controla por características sociodemográficas. Este incluye tres variables binarias relacionadas con haber asistido a una secundaria privada, ser mujer y vivir actualmente con su pareja; $y$ una variable continua que controla por el nivel de desarrollo, medido a través del índice de competitividad cantonal. Estas son variables de control relativamente estándar en la literatura. El tipo de escuela o colegio al que se asiste puede servir para proveer información sobre el contexto de la persona (Chevalier, 2011). La variable asociada a ser mujer y vivir actualmente con la pareja pueden capturar algunas características asociadas a los roles de género y maternidad, mientras que el índice de competitividad cantonal puede capturar características asociadas con el ambiente y que usualmente se asocian a la zona o región ${ }^{13}$. Este último modelo se estimó para mujeres, hombres y ambos grupos considerados en conjunto.

Con el fin de medir cuánto del diferencial de empleo puede explicarse por la discriminación, se estimó una descomposición Blinder-Oaxaca para modelos no lineales utilizando la metodología y el comando nldecompose de Stata de Bauer y Sinning (2008) y Sinning, Hahn y Bauer (2008), respectivamente. El diferencial en el empleo promedio $(\bar{e})$ observado entre dos grupos (g), hombres $(m)$ y mujeres $(f)$, está dado por las diferencias entre características individuales $\left(x_{i, g}\right)$ y las diferencias entre coeficientes $(\beta)$ asociados a cada grupo, tal que:

$$
\begin{gathered}
\bar{e}_{m}-\bar{e}_{f}=\left\{E_{\beta^{*}}\left(e_{i m} \mid x_{i m}\right)-E_{\beta^{*}}\left(e_{i f} \mid x_{i f}\right)\right\}+\left\{E_{\beta_{m}}\left(e_{i m} \mid x_{i m}\right)-E_{\beta^{*}}\left(e_{i f} \mid x_{i f}\right)\right\} \\
+\left\{E_{\beta^{*}}\left(e_{i f} \mid x_{i f}\right)-E_{\beta_{f}}\left(e_{i f} \mid x_{i f}\right)\right\}
\end{gathered}
$$

donde $\beta^{*}$ refiere al vector de coeficientes contrafácticos en ausencia de discriminación, el cual se estima a partir de un modelo agregado entre los grupos, tal y como lo sugieren Oaxaca y Ransom (1994).

\section{RESULTADOS}

La tabla 2 presenta los efectos marginales medios para los modelos. Todos los modelos son diferentes a un modelo sin regresores, con valores-p para la prueba $\mathrm{F}$ de significancia del modelo iguales 0 menores a 0.0002 . Los resultados del modelo A sugieren que estudiar un programa predominantemente masculino incrementa, en promedio, en 1.3 p.p. la probabilidad de empleo relativa a aquellas personas que optaron por un programa en una disciplina integrada. En contraste, estudiar una disciplina dominada por mujeres disminuye, en promedio, la probabilidad de empleo respecto al grupo de referencia en 1.3 p.p., al nivel de confianza estándar. Esto podría sugerir la existencia de un premio asociado a las disciplinas masculinas más tradicionales, tal y como lo apunta la teoría de la polución de Goldin (2002). Al nivel de confianza estándar, la probabilidad de empleo no se asocia con el área de estudio. Esto podría sugerir que la segregación de género es crucial para comprender el empleo de las personas graduadas, mas no así la disciplina estudiada.

Controlar por otras características asociadas al capital humano modifica los resultados ligeramente: en este escenario, el castigo por estudiar disciplinas predominantemente femeninas desaparece, dejando únicamente el premio para aquellas personas con un título en una disciplina

12 Véase, por ejemplo, Rosenfeld, 1979; Oaxaca, 1973; Goldin, 1983; Oaxaca y Ransom, 1994; Blau y Kahn, 2007; Black, et al., 2008; Manning y Swaffield, 2008; Mumford y Smith, 2009; Cedeño, González y Pizarro, 2015; y Rodríguez y Segura, 2015.

13 Véase por ejemplo, Becker, 1971; Oaxaca, 1973; Oaxaca y Ransom, 1994; Machin y Puhani, 2002, 2005; Black, et al., 2008; Castro, et al., 2014; Cedeño, et al., 2015; Ortega Masagué, 2008 y Rodríguez y Segura, 2015. 
dominada por hombres de aproximadamente 1.6 p.p. relativo a aquellas personas en una disciplina integrada. No obstante, la probabilidad de empleo es menor para quienes obtuvieron un título en ciencias sociales, recursos naturales e ingeniería relativa a aquellas personas en disciplinas económicas. De hecho, las tasas de desempleo superan el 3\% para todas las ciencias sociales con excepción de archivística, producción audiovisual y derecho, con la mayor tasa de desempleo en trabajo social $(8.2 \%)$, ciencias políticas $(7.1 \%)$ y relaciones públicas (7\%). La tasa de desempleo para personas graduadas de recursos naturales es de $3.7 \%$, es decir, 0.7 p.p. por encima de la tasa para todas las personas graduadas y es mayor para aquellas personas en fitotecnia (10.3\%), ingeniería de administración agrícola (9.6\%) y geografía (8\%). Respecto a ingeniería, inicialmente pareciera contradictorio encontrar una relación negativa con el empleo, dado que es un área dominada por hombres. De hecho, la mayoría de disciplinas en esta área presentan tasas de desempleo inferiores a 1.5\%. Pero, dentro de ellas, las personas que se encuentran desempleadas están altamente concentradas en arquitectura y diseño de interiores, con tasas de desempleo de 5.7\% y 11\%, respectivamente. La primera refiere a una disciplina integrada, mientras la última está dominada por mujeres. En contraste, las ingenierías más tradicionales, tales como topografía, ingeniería eléctrica o civil muestran tasas de desempleo extremadamente bajas.

Como es de esperar, los resultados del modelo B también aportan evidencia de que años adicionales de escolaridad, ya sea a través de una licenciatura o de un segundo diploma, se encuentran positiva y significativamente asociados con el empleo, lo cual es consistente con la teoría del capital humano. En forma similar, la experiencia profesional potencial parece estar positiva y decrecientemente asociada al empleo, no así la variable asociada a la experiencia no profesional potencial. Esto es esperable, pues las capacidades desarrolladas para ejercer un empleo profesional no necesariamente estarán vinculadas con aquellas capacidades que podrían acumularse en empleos de menor cualificación, como los que puede aspirar una persona que estudie y trabaje a la vez. La información no provee evidencia a favor de la creencia popular de que las graduadas de universidades públicas sean más demandadas en el mercado laboral costarricense, ya que graduarse de una universidad pública no se asocia con mayores probabilidades de empleo.

Agregar variables socio-demográficas (modelo C) no altera la significancia o magnitud de los efectos marginales discutidos anteriormente. El tipo de secundaria es una variable utilizada para aproximar el contexto socio-económico y resulta positiva y significativamente asociada con el empleo: asistir a una secundaria privada incrementa, en promedio, la probabilidad de empleo en 1.1 p.p. Esto podría sugerir la existencia de capital cultural expresado a través de diferencias de clase en términos del acceso al empleo y oportunidades en el mercado laboral, oportunidades para generar redes y establecer contactos. También podría señalar diferencias en la calidad educativa, pero esto es menos probable, ya que no hay evidencia de que las personas provenientes de secundarias privadas tengan un mejor desempeño académico que aquellas provenientes de instituciones públicas (Abarca, Leiva, Robalino \& Saborío, 2016).

Los resultados también muestran que vivir con una pareja y ser mujer se encuentran negativamente asociadas al empleo a un nivel de confianza de 90\%. Estas variables podrían estar capturando los efectos de la información faltante en la encuesta sobre las decisiones reproductivas de las mujeres. Podría ser que las personas graduadas que viven con su pareja sean más proclives a reproducirse $y$, por tanto, enfrenten restricciones asociadas a las dificultades de encontrar un empleo que empate sus necesidades por horarios flexibles, contratos de tiempos parciales y políticas más amigables a la familia en general. La variable que controla por el género podría también estar capturando alguna de esta información faltante, ya que las mujeres aún son las principales responsables de las tareas domésticas en Costa Rica. Esta variable también podría estar capturando el efecto de variables institucionales asociadas a las diferencias con que la sociedad trata a mujeres $y$ hombres. 
TABLA 2

EFECTOS MARGINALES MEDIOS DE LA PROBABILIDAD DE EMPLEO DE LAS PERSONAS GRADUADAS DE UNIVERSIDAD

\begin{tabular}{|c|c|c|c|c|c|}
\hline Calidad de la educación & Modelo A & Modelo B & Modelo C: & Modelo C: & Modelo C: \\
\hline & & & Agregado & Femenino & Masculino \\
\hline \multicolumn{6}{|l|}{$\begin{array}{l}\text { Segregación sexual (referencia=disciplinas } \\
\text { integradas) }\end{array}$} \\
\hline \multirow[t]{2}{*}{ Disciplinas predominantemente masculinas } & $0.013^{* * * *}$ & $0.016^{* * * *}$ & $0.015^{* * * *}$ & $0.028 * * *$ & 0.004 \\
\hline & $(0.004)$ & $(0.004)$ & $(0.005)$ & $(0.007)$ & $(0.004)$ \\
\hline \multirow[t]{2}{*}{ Disciplinas predominantemente femeninas } & $-0.013 * *$ & -0.008 & -0.003 & 0.001 & $-0.010^{*}$ \\
\hline & $(0.006)$ & $(0.006)$ & $(0.005)$ & $(0.007)$ & $(0.006)$ \\
\hline \multicolumn{6}{|l|}{$\begin{array}{l}\text { Área de estudio (referencia = disciplinas } \\
\text { económicas): }\end{array}$} \\
\hline \multirow[t]{2}{*}{ Humanidades } & -0.006 & -0.004 & -0.008 & -0.005 & -0.011 \\
\hline & $(0.012)$ & $(0.008)$ & $(0.008)$ & $(0.010)$ & $(0.009)$ \\
\hline \multirow[t]{2}{*}{ Ciencias } & $-0.025^{*}$ & -0.017 & -0.022 & 0.002 & $-0.027 *$ \\
\hline & $(0.014)$ & $(0.012)$ & $(0.014)$ & $(0.018)$ & $(0.014)$ \\
\hline \multirow[t]{2}{*}{ Ciencias sociales } & -0.008 & $-0.010^{*}$ & $-0.013 * * *$ & $-0.016^{* *}$ & -0.007 \\
\hline & $(0.007)$ & $(0.005)$ & $(0.005)$ & $(0.007)$ & $(0.006)$ \\
\hline \multirow[t]{2}{*}{ Educación } & 0.001 & -0.005 & -0.003 & -0.005 & 0.002 \\
\hline & $(0.007)$ & $(0.006)$ & $(0.006)$ & $(0.008)$ & $(0.006)$ \\
\hline \multirow[t]{2}{*}{ Recursos naturales } & $-0.027^{*}$ & $-0.030^{* *}$ & $-0.032 * *$ & $-0.038 * * *$ & -0.029 \\
\hline & $(0.014)$ & $(0.013)$ & (0.013) & $(0.014)$ & $(0.021)$ \\
\hline \multirow[t]{2}{*}{ Ingeniería } & $-0.020^{*}$ & $-0.023^{* *}$ & $-0.031^{* *}$ & $-0.053^{* *}$ & -0.016 \\
\hline & $(0.011)$ & $(0.011)$ & (0.013) & $(0.026)$ & $(0.011)$ \\
\hline \multirow[t]{2}{*}{ Ciencias de la salud } & 0.012 & 0.007 & 0.005 & 0.004 & 0.008 \\
\hline & $(0.008)$ & $(0.008)$ & $(0.009)$ & $(0.011)$ & $(0.007)$ \\
\hline \multirow[t]{2}{*}{ Obtener una licenciatura } & & $0.023^{* * *}$ & $0.024 * * *$ & $0.028 * * *$ & $0.015^{* * *}$ \\
\hline & & $(0.005)$ & $(0.005)$ & $(0.007)$ & $(0.003)$ \\
\hline \multirow{2}{*}{\multicolumn{2}{|c|}{ Haberse graduado de una universidad pública }} & 0.005 & 0.005 & 0.002 & $0.008^{* *}$ \\
\hline & & $(0.005)$ & $(0.005)$ & $(0.007)$ & $(0.004)$ \\
\hline \multirow[t]{2}{*}{ Obtener un segundo título } & & $0.014^{* * *}$ & $0.014^{* * *}$ & $0.020 * * *$ & 0.004 \\
\hline & & $(0.004)$ & $(0.004)$ & $(0.005)$ & $(0.004)$ \\
\hline \multicolumn{6}{|l|}{ Experiencia potencial } \\
\hline \multirow[t]{2}{*}{ Años entre graduación universitaria y secundaria } & & 0.000 & 0.000 & $0.001^{*}$ & -0.000 \\
\hline & & $(0.000)$ & $(0.000)$ & $(0.000)$ & $(0.000)$ \\
\hline \multirow[t]{2}{*}{ Años transcurridos desde la graduación } & & $0.016^{* * * *}$ & $0.016^{* * *}$ & $0.016^{* * * *}$ & $0.014^{*}$ \\
\hline & & $(0.004)$ & $(0.004)$ & $(0.005)$ & $(0.007)$ \\
\hline \multirow{2}{*}{$\begin{array}{l}\text { Años transcurridos desde la graduación al } \\
\text { cuadrado }\end{array}$} & & $-0.001^{* *}$ & $-0.001^{* *}$ & -0.001 & -0.001 \\
\hline & & $(0.000)$ & $(0.000)$ & $(0.000)$ & $(0.001)$ \\
\hline
\end{tabular}




\begin{tabular}{|c|c|c|c|c|c|}
\hline & Modelo A & Modelo B & Modelo C: & Modelo C: & Modelo C: \\
\hline & & & Agregado & Femenino & Masculino \\
\hline \multicolumn{6}{|l|}{ Variables de control } \\
\hline \multirow[t]{2}{*}{ Haber asistido a una secundaria privada } & & & $0.011^{* * *}$ & $0.013 * *$ & $0.005^{*}$ \\
\hline & & & $(0.004)$ & $(0.006)$ & $(0.003)$ \\
\hline \multirow[t]{2}{*}{ Vivir con una pareja } & & & $-0.008^{*}$ & $-0.013 * *$ & 0.004 \\
\hline & & & $(0.004)$ & $(0.006)$ & $(0.005)$ \\
\hline \multirow[t]{2}{*}{ Índice de desarrollo local } & & & 0.001 & 0.003 & -0.001 \\
\hline & & & $(0.007)$ & $(0.010)$ & $(0.008)$ \\
\hline \multirow[t]{2}{*}{ Ser mujer } & & & $-0.018^{* * *}$ & & \\
\hline & & & $(0.004)$ & & \\
\hline Observaciones & 22308 & 22308 & 22308 & 13218 & 9090 \\
\hline Prob $>$ F of para la regresión logit & 0.0002 & 0.0000 & 0.0000 & 0.0000 & 0.0000 \\
\hline
\end{tabular}

$* \mathrm{p}<0.10, * * \mathrm{p}<0.05, * * * \mathrm{p}<0.01$

Fuente: Estimaciones propias con datos de CONARE (2013, 2015).

Como ser mujer reduce la probabilidad de empleo en casi 2 puntos porcentuales, se procedió a estimar el modelo en forma separada para mujeres y hombres, con el fin de revelar mejor las diferencias entre los géneros. Las columnas D y E muestran evidencia adicional de que la segregación sexual de las disciplinas afecta el empleo de mujeres y hombres en forma diferente. Mientras las mujeres parecieran disfrutar un premio por transgredir los espacios masculinos, a los hombres que transgreden los espacios dominados por las mujeres se les castiga. Para las mujeres, esto recuerda la hipótesis de Gladwell (2013) sobre los trofeos femeninos, según la cual a las mujeres pioneras que incursionan en carreras dominadas por hombres se les premia, porque se les percibe como excepcionales. Pero, el hecho de que los hombres tengan una menor probabilidad de empleo si estudiaron una disciplina dominada por mujeres podría estar señalizando un alto costo por transgredir la masculinidad. Para analizar la evidencia que podría apoyar la teoría de la polución de Goldin (2002) se debe observar las diferencias al interior de las áreas de estudio: mientras el área de estudio no es significativa para explicar el empleo masculino, las mujeres en recursos naturales, ciencias sociales e ingeniería tienen una menor probabilidad de estar empleadas relativa a quienes se graduaron de una disciplina económica. Lo anterior puede sugerir que las mujeres en estas áreas son más vulnerables a la discriminación durante los procesos de contratación. De hecho, la proporción de mujeres desempleadas en estas áreas es más del doble que sus contrapartes masculinos, con tasas de desempleo femenino alrededor o superiores al 5\% en todos estos casos. Esto también resuena con el argumento de Power (1975) sobre la discriminación en contra de las mujeres y la preferencia por los hombres. Igualmente, resulta significativo que las variables asociadas con la cantidad del capital humano, i.e. los años de escolaridad y la experiencia, sean considerablemente más significativas al explicar el empleo femenino, como si se demandara más de las mujeres para ser contratadas.

Para las mujeres, la clase socio-económica podría ayudar a contrarrestar algunas de las desventajas asociadas a la sexuación de las disciplinas de estudio: haber asistido a una secundaria privada aumenta la probabilidad de estar empleada en 1.3 p.p. al nivel de confianza estándar. 
En contraste, vivir con su pareja disminuye la probabilidad de empleo en casi la misma magnitud. El hecho de que esta variable sea significativa únicamente para explicar el empleo femenino apoya los argumentos previos de que podría estar capturando los efectos de alguna de la información faltante sobre las decisiones reproductivas. Finalmente, el contexto socioeconómico no resultó significativo para explicar el empleo.
La tabla 3 presenta los resultados de la descomposición Blinder-Oaxaca para modelos no lineales y muestra que únicamente alrededor de una cuarta parte del diferencial observado es explicado por las características individuales, mientras que $46.1 \%$ de él obedece a la ventaja masculina y el restante $28.4 \%$ se explica por la desventaja femenina. Es decir, tres cuartas partes del diferencial de empleo entre mujeres $y$ hombres se explica por el coeficiente de discriminación (diferencia en retornos).

TABLA 3

DESCOMPOSICIÓN OAXACA-BLINDER ENTRE EMPLEO MASCULINO Y FEMENINO DE PERSONAS GRADUADAS DE LA UNIVERSIDAD

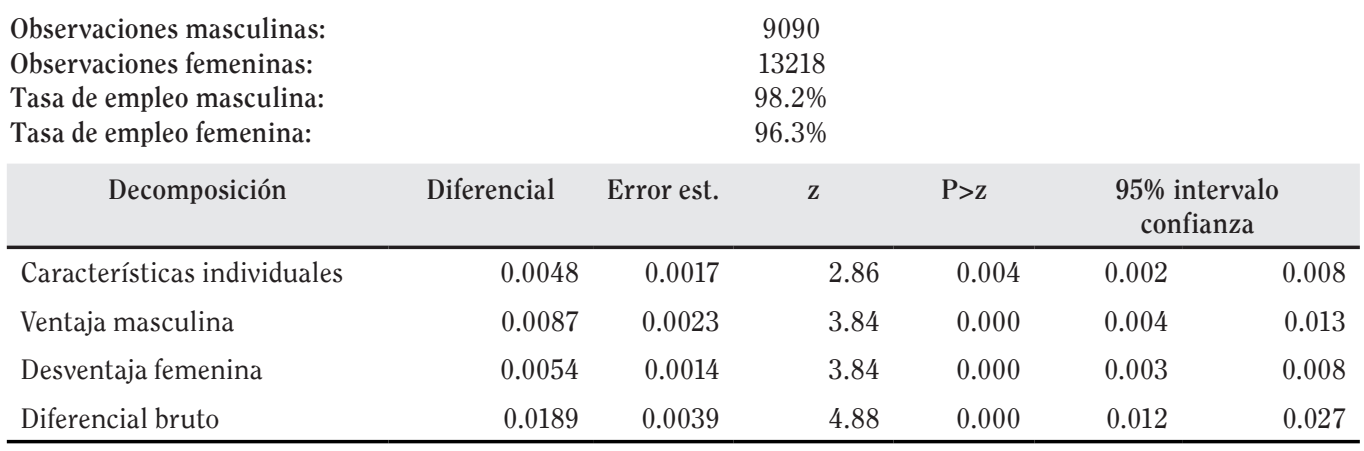

Fuente: Estimaciones propias con datos de CONARE (2013, 2015).

\section{CONCLUSIONES}

Los principales resultados de este artículo proveen información que apoya que la segregación sexual de las disciplinas de estudio se encuentra fuertemente relacionada a los resultados de empleo para las personas graduadas de la universidad. A pesar de que la mayor parte de quienes se gradúan de la universidad son mujeres, las disciplinas de estudio se hallan segregadas por género: casi la mitad de las disciplinas en las bases de datos analizadas se catalogaron como predominantemente femeninas, con las mujeres mayormente concentradas en educación. Las disciplinas dominadas por hombres parecen ser más exclusivas y están asociadas a mejores oportunidades de empleo, pero apenas representan $17 \%$ de todas las disciplinas. Estas se encuentran también más entrelazadas con características más enraizadas en la concepción de la masculinidad tales como la tosquedad, fuerza física (Carrigan, Connell \& Lee, 1985) y habilidad matemática (Levy, 1972), la cual puede asociarse con disciplinas como topografía, ingeniería civil o mecánica. El hecho de que la mayor parte de las personas graduadas sean mujeres puede también explicar por qué el número de disciplinas predominantemente masculinas sea pequeño relativo al número de disciplinas integradas o predominantemente femeninas. Una gran cantidad de las disciplinas tradicionalmente asociadas a los hombres, tales como química, derecho, economía o medicina, ahora muestran paridad de género y son, por tanto, clasificadas como integradas. Tal integración de género puede ser producto de la reversión 
de género en educación experimentada en los años noventa: conforme las mujeres dominen la educación terciaria, irán derribando los límites impuestos por las esferas separadas. No obstante, sí pareciera que las mujeres son más cautas que los hombres en hacer esto: mientras los hombres se encuentran más dispersos entre las diferentes disciplinas, la mayoría de mujeres parece optar por disciplinas que refuerzan la segregación de género. La persistencia de esta segregación, contrarrestada por el número creciente de mujeres que entran y se gradúan de la universidad, resultó en un índice de disimilitud de 31\%, que puede ser considerado moderado.

Los resultados también indican que la calidad del título obtenido (medido por el área de estudio, el grado y el sector universitario) se asocia con la probabilidad de encontrar empleo posteriormente en el mercado laboral. Las personas graduadas de disciplinas predominantemente masculinas son más propensas a estar empleadas que quienes obtuvieron un título de una disciplina integrada. En particular, las mujeres graduadas de disciplinas predominantemente masculinas tienen mayor probabilidad de estar empleadas, tal vez en parte porque sean percibidas como excepcionales por transgredir estos espacios masculinos. Al contrario, los hombres que incursionan en carreras dominadas por mujeres cuentan con una menor probabilidad de estar empleados, lo que podría sugerir un mayor costo por retar la masculinidad en una sociedad patriarcal y latina. La teoría de la polución de Goldin (2002), según la cual a las mujeres que incursionan en carreras masculinas se les excluye porque ponen en riesgo el estatus social de la ocupación y para evadir esta exclusión se termina por crear nuevas y segregadas ocupaciones, puede servir como una explicación para comprender por qué las mujeres que incursionan en carreras como recursos naturales e ingeniería enfrentarían mayores riesgos de desempleo comparado con aquellas que optan por una carrera en alguna disciplina económica que es más integrada. En forma similar, una preferencia por los hombres puede explicar por qué las mujeres en las ciencias sociales enfrentan un mayor riesgo de desempleo, pero los hombres no.

Los resultados también muestran una mayor relación entre el empleo y los años de escolaridad (medidos a través del grado obtenido) y la experiencia potencial como profesional para las mujeres que para los hombres. Esto último sugiere que las mujeres que han invertido más en capital humano tienen mayor probabilidad de tener arraigo al mercado laboral y, por tanto, de estar empleadas, que aquellas mujeres graduadas que únicamente obtuvieron un título de bachillerato. Asimismo, haber asistido a una secundaria privada, que usualmente se asocia con un contexto familiar de mayor clase social, se encuentra positivamente asociado con que las mujeres estén empleadas. Esto sugiere que la clase social puede ser un factor importante para comprender la accesibilidad y movilidad en el mercado laboral. Finalmente, la mayor limitación del estudio es la falta de disponibilidad de información concerniente a las decisiones reproductivas, las cuales sabemos son esenciales para comprender el empleo y participación laboral femenina. Esto apunta a la necesidad de mejorar la recolección de información en los países en vías de desarrollo.

En síntesis, los resultados advierten sobre la persistencia de los efectos de la segregación de género en el mercado laboral, en este caso, respecto a la sexuación de disciplinas de estudio de la población profesional. Pero los datos también permiten una lectura optimista, al sugerir que el mayor porcentaje de mujeres graduadas universitarias pueda, eventualmente, derribar barreras hacia un mundo más integrado.

\section{REFERENCIAS}

Abarca, A., Leiva, R., Robalino, J., \& Saborío, M. (2016). Diferencias en la permanencia y el desempeño en la educación superior entre estudiantes provenientes de colegios públicos y privados. (Serie de documentos de trabajo 16-03), San José: Escuela de Economía, Universidad de Costa Rica. Recuperado de http://economia.ucr.ac.cr/sites/default/files/files/ EE\%20UCR\%20SDT\%2016-03.pdf 
Adsera, A. (2005). Vanishing Children: From High Unemployment to Low Fertility in Developed Countries. American Economic Review, 95(2), 189-193. https://doi. org/10.1257/000282805774669763

Ahn, N. \& Mira, P. (2002) A note on the changing relationship between fertility and female employment rates in developed countries. Journal of Population Economics, 15(4), 667-682. https://doi.org/10.1007/s001480100078

Azmat, G., Güell, M., \& Manning, A. (2006). Gender Gaps in Unemployment Rates in OECD Countries. Journal of Labor Economics, 24(1), 1-37. https://doi.org/10.1086/497817

Bauer, T. K., \& Sinning, M. (2008). An extension of the Blinder-Oaxaca decomposition to nonlinear models. AStA Advances in Statistical Analysis, 92(2), 197-206. https://doi.org/10.1007/s10182008-0056-3

Becker, G. S. (1962). Investment in Human Capital: A Theoretical Analysis. Journal of Political Economy, 70(5, Part 2), 9-49. https://doi.org/10.1086/258724

Becker, G.S. (1971). The Economics of discrimination. Chicago, IL: The University of Chicago Press.

Becker, G.S. (1991). Treatise on the family. Massachusetts: Harvard University Press.

Black, D.A., Haviland, A.D.; Sanders, S.G. \& Taylor, L.J. (2008). Gender wage disparities among the highly educated. The Journal of Human Resources, 43(3), 630-659. https://doi.org/10.3368/ jhr.43.3.630

Blau, F.D. (Invierno, 1999). Women's economic well-being, 1970-1995: Indicators and trends. Focus, 20(1), 4-10.

Blau, F. D., \& Kahn, L. M. (2007). The Gender Pay Gap: Have Women Gone as Far as They Can? Academy of Management Perspectives, 21(1), 7-23. https://oi.org/10.5465/ AMP.2007.24286161

Carrigan, T., Connell, B., \& Lee, J. (1985). Toward a new sociology of masculinity. Theory and Society, 14(5), 551-604. https://doi.org/10.1007/BF00160017

Castro, J. M., Garita, J., \& Odio, M. (2014). Análisis sobre la dinámica de transición y duración del desempleo en Costa Rica. Revista de Ciencias Económicas, 32(2), 39. https://doi.org/10.15517/ rce.v32i2.17251

Cedeño, J., González, A. \& Pizarro, K. (2015). Discriminación salarial por género en Costa Rica durante los ciclos económicos del período 1992-2013. (Seminario de graduación para optar por el grado de Licenciatura) San José, Universidad de Costa Rica. Recuperado de https://hdl. handle.net/10669/29982

Chevalier, A. (2011). Subject choice and earnings of UK graduates. Economics of Education Review, 30(6), 1187-1201. https://doi.org/10.1016/j.econedurev.2011.04.007

Consejo Nacional de Rectores (2013). Seguimiento de la condición laboral de las personas graduadas 2000-2007 de las universidades costarricenses. San José: CONARE.

Consejo Nacional de Rectores (2015). Seguimiento de la condición laboral de las personas graduadas 2008-2010 de las universidades costarricenses. San José: CONARE. Recuperado de https:// www.conare.ac.cr/servicios/category/33-observatorio-de-profesiones?download=77:seguim iento-de-la-condicion-laboral-de-las-personas-graduadas-2008-2010-de-las-universidadescostarricenses

Darity Jr., W.A. \& Mason, P.L. (1998). Evidence on discrimination in employment: codes of color, codes of gender. The Journal of Economic Perspectives, 12(2), 63-90. https://doi.org/10.1257/ jep.12.2.63

Gladwell, M. (2013). Tokens, pariahs and pioneers. (Video). Recuperado de https://www.youtube. $\mathrm{com} /$ watch?v=rW888dolawo.

Goldin, C. (1983) Life-cycle labor force participation of married women: historical evidence and implications. (Documento de trabajo 1251). National Bureau of Economic Research. https:// doi.org/10.3386/w1251 
Goldin, C. (2002). A pollution theory of discrimination: male and female differences in occupations and earnings. (Documento de trabajo 8985). National Bureau of Economic Research Working Paper. https://doi.org/10.3386/w8985

Goldin, C. \& Katz, L. (2012). The most egalitarian of all professions: pharmacy and the evolution of a family-friendly occupation. (Documento de trabajo 18410). National Bureau of Economic Research. https://doi.org/10.3386/w18410

Gutiérrez, I. \& Kikut, L. (2014). Personas graduadas en el período 2008-2010 de universidades costarricenses. San José: Estado de la Educación.

Hakim, C. (2002). Lifetime preferences as determinants of women's differentiated labor market careers. Work and Occupations, 29(4), 428-459. https://doi. org/10.1177/0730888402029004003

Heckman J.J. (1976). The common structure of statistical models of truncation, sample selection and limited dependent variables and a simple estimator for such models. Annals of Economic and Social Measurement, 5(4), 475-492. Recuperado de https://www.nber.org/chapters/c10491

Heckman, J.J. \& Willis, R.J. (1977). A beta-logistic model for the analysis of sequential labor force participation by married women. Journal of Political Economy, 85 (1), 27-58. https://doi. org/10.1086/260544

Levy, B. (1972). The school's role in the sex-role stereotyping of girls: a feminist review of the literature. Feminist studies, 1(1), 5-23. https://doi.org/10.2307/3180104

Machin, S. \& Puhani, P. (2002). Subject of degree and the gender wage differential. Evidence from the UK and Germany. (Documento de trabajo IZA 553). Institute for the Study of Labor.

Machin, S. \& Puhani, P. (2005). The contribution of degree subject to the gender wage gap for graduates. Preliminary draft. Presentado en Annual Meeting of the Swiss Society of Economics and Statistics on Resource Economics, Technology and Sustainable Development, Suiza.

Manning, A., \& Swaffield, J. (2008). The gender gap in early-career wage growth. The Economic Journal, 118(530), 983-1024. https://doi.org/10.1111/j.1468-0297.2008.02158.x

Miech, R. A., Eaton, W., \& Liang, K.-Y. (2003). Occupational Stratification Over the Life Course: A Comparison of Occupational Trajectories Across Race and Gender During the 1980s and 1990s. Work and Occupations, 30(4), 440-473. https://doi.org/10.1177/0730888403256459

Mincer, J. (1974) Schooling, experience and earnings. New York: Columbia University Press.

Ministerio de Educación Pública (MEP), Dirección de Planificación Institucional, Departamento de Análisis Estadístico. (2016). Matrícula inicial en III ciclo y educación diversificada, diurna y nocturna por año cursado y sexo, según zona y dependencia, año 2015. Recuperado de http:// www.mep.go.cr/indicadores_edu/cifras_zona_dependencia3.html.

Mumford, K., \& Smith, P. N. (2009). What determines the part-time and gender earnings gaps in Britain: evidence from the workplace. Oxford Economic Papers, 61(Supplement 1), i56-i75. https://doi.org/10.1093/oep/gpn041

Nagore-García, A. (2015). Gender differences in unemployment dynamics and initial wages over the business cycle. Valencia: Department of Applied Economics, University of Valencia. Recuperado de http://www.uv.es/esteco/Seminaris_2015_Ponencies/2015-11-Juny_Nagore_ Amparo.pdf.

Oaxaca, R. (1973). Male-Female Wage Differentials in Urban Labor Markets. International Economic Review, 14(3), 693-709. https://doi.org/10.2307/2525981

Oaxaca, R. L., \& Ransom, M. R. (1994). On discrimination and the decomposition of wage differentials. Journal of Econometrics, 61(1), 5-21. https://doi.org/10.1016/03044076(94)90074-4

Organización Internacional del Trabajo (2014). Global Employment Trends 2014: Risk of a jobless recovery? Ginebra: Organización Internacional del Trabajo. 
Ortega-Masagué, A.C. (2008). Gender gaps in unemployment rates in Argentina. Económica, LIV(12), 161-202. Recuperado de http://www.economica.econo.unlp.edu.ar/wp/?p=5124

Power, M. (1975). Woman's Work Is Never Done- by Men: A Socio-Economic Model of SexTyping in Occupations. Journal of Industrial Relations, 17(3), 225-239. https://doi. org/10.1177/002218567501700301

Rodríguez, M. \& Segura, M. (2015). ¿Existe un techo de cristal en la distribución salarial femenina en Costa Rica? (Memoria de seminario de graduación para optar por el grado de Licenciatura). San José, Universidad de Costa Rica. Revuperado de http://repositorio.ciem. ucr.ac.cr/jspui/handle/123456789/171

Rosenfeld, R. A. (1979). Women's occupational careers. Individual and structural explanations. Sociology of Work and Occupations, 6(3), 283-311. https://doi. org/10.1177/073088847900600302

Sinning, M., Hahn, M. \& Bauer, T.K. (2008). The Blinder-Oaxaca decomposition for nonlinear regression models. The Stata Journal, 8(4), 480-492. Recuperado de http://www.statajournal.com/article.html?article=st0152

Sullivan, A., Joshi, H. \& Leonard, D. (2008). Single-sex schooling and academic attainment at school and through the life course, CLS working paper 2008/3. London: Centre for Longitudinal Studies.

Walby, S. (1988). "Segregation in employment in social and economic theory". En S., Walby, (Ed.). Gender segregation at work. (pp. 1-28). Milton Keynes: Open University Press.

Ulate, A., Madrigal, G., Ortega, R. \& Jiménez, E. (2012). Índice de competitividad cantonal. Costa Rica 2006-2011. San José: Universidad de Costa Rica, Observatorio del Desarrollo.

Banco Mundial (2015). World Development Indicators. Recuperado de: http://databank.worldbank. org/data/reports.aspx?source=world-development-indicators

Zafar, B. (2013). College Major Choice and the Gender Gap. Journal of Human Resources, 48(3), 545-595. https://doi.org/10.3368/jhr.48.3.545 


\section{ANEXO 1: \\ DISCIPLINAS DE ESTUDIO POR SEGREGACIÓN DE GÉNERO}

\begin{tabular}{|c|c|}
\hline \multicolumn{2}{|c|}{ Disciplinas predominantemente masculinas } \\
\hline Filosofía & Geografía \\
\hline Teología & Ingeniería civil \\
\hline Física & Topografía \\
\hline Ingeniería informática & Ingeniería industrial \\
\hline Administración agrícola & Ingeniería mecánica \\
\hline Educación musical & Ingeniería eléctrica \\
\hline Educación agrícola & Ingeniería de materiales \\
\hline Agronomía & Ingeniería agrícola \\
\hline Fitotecnia & \\
\hline \multicolumn{2}{|c|}{ Disciplinas integradas } \\
\hline Diseño gráfico & Educación en ciencias \\
\hline Arte publicitario & Educación en matemáticas \\
\hline Geología & Educación industrial técnica \\
\hline Estadística & Biotecnología \\
\hline Química & Economía agrícola \\
\hline Técnica de laboratorio clínico & Zootecnia \\
\hline Administración pública & $\underline{\text { Silvicultura y ecología }}$ \\
\hline Contabilidad & Arquitectura \\
\hline Finanzas & Ingeniería química \\
\hline Mercadeo & Seguridad laboral \\
\hline Antropología & Diseño industrial \\
\hline Producción audiovisual & Ingeniería electromédica \\
\hline Ciencias políticas & Medicina \\
\hline Derecho & Imagenología \\
\hline Economía & Veterinaria \\
\hline Historia & Educación general \\
\hline \multicolumn{2}{|l|}{ Ciencias forenses } \\
\hline \multicolumn{2}{|c|}{ Disciplinas predominantemente femeninas } \\
\hline Artes dramáticas & Profesora de inglés \\
\hline Arte & Profesora de francés \\
\hline Danza & Profesora de psicología y filosofía \\
\hline Literatura y lingüística & Profesora de computación \\
\hline Francés & Orientación \\
\hline Inglés & Profesora de artes \\
\hline Bibliotecología & Educación para el hogar \\
\hline Biología & Profesora de religión \\
\hline
\end{tabular}




\section{ANEXO 1: \\ DISCIPLINAS DE ESTUDIO POR SEGREGACIÓN DE GÉNERO}

\begin{tabular}{|c|c|}
\hline \multicolumn{2}{|c|}{ Disciplinas predominantemente femeninas } \\
\hline Recursos humanos & Educación especial \\
\hline Administración de recursos de salud & Administración educativa \\
\hline Archivística & Educación técnica en servicios \\
\hline Secretariado & Tecnología de alimentos \\
\hline Periodismo & Diseño de interiores \\
\hline Publicidad & Terapia física \\
\hline Relaciones públicas & Terapia ocupacional \\
\hline Relaciones internacionales & Terapia respiratoria \\
\hline Psicología & Información de la salud y registros médicos \\
\hline Sociología & Audiología \\
\hline Trabajo social & Nutrición \\
\hline Secretariado & Tecnología de alimentos \\
\hline Periodismo & Diseño de interiores \\
\hline Publicidad & Terapia física \\
\hline Relaciones públicas & Terapia ocupacional \\
\hline Relaciones internacionales & Terapia respiratoria \\
\hline Psicología & $\begin{array}{l}\text { Información de la salud y registros } \\
\text { médicos }\end{array}$ \\
\hline Sociología & Audiología \\
\hline Trabajo social & Nutrición \\
\hline Planificación & Optometría \\
\hline Turismo & Salud pública \\
\hline Educación (general) & Odontología \\
\hline Educación preescolar & Farmacia \\
\hline Inglés para educación preescolar & Microbiología \\
\hline Educación primaria & Enfermería \\
\hline Inglés para educación primaria & Otras tecnologías \\
\hline Profesora de español & Otras tecnologías \\
\hline
\end{tabular}

